\title{
A New Method of Voiced/Unvoiced Classification Based on Clustering
}

\author{
Mojtaba Radmard, Mahdi Hadavi, Mohammad Mahdi Nayebi
}

Department of Electrical Engineering, Sharif University of Technology, Tehran, Iran. Email: \{radmard, mahdi_hadavi\}@ee.sharif.ir, nayebi@sharif.ir

Received June $2^{\text {nd }}, 2011$; revised October $14^{\text {th }}, 2011$; accepted October $23^{\text {rd }}, 2011$.

\begin{abstract}
In this paper, a new method for making v/uv decision is developed which uses a multi-feature v/uv classification algorithm based on the analysis of cepstral peak, zero crossing rate, and autocorrelation function (ACF) peak of short-time segments of the speech signal by using some clustering methods. This v/uv classifier achieved excellent results for identification of voiced and unvoiced segments of speech.
\end{abstract}

Keywords: Speech, Voiced, Unvoiced, Clustering, Cepstrum, Autocorrelation, Zero Crossing

\section{Introduction}

The voiced/unvoieced decision is critical in many speech analysis/synthesis systems because it is essential to know whether the speech production system involves vibration of the vocal cords [1-4]. This decision is required for many applications, including modeling for analysis/synthesis, detection of model changes for segmentation purposes and signal characterization for indexing and recognition applications [1]. The periodicity of this vibration makes the voiced segments periodic and so distinguishable from the noisy-like unvoiced segments [5]. Since the speech signals are quasi-periodic [6-9], making the decision gets hard. Other difficulties are represented in $[3,10]$.

Common methods extract a feature from speech segments and make the v/uv decision according to whether the value of the feature is above or below a pre-determined threshold. The feature can be the cepstral peak $[6,11]$, some mel-frequency cepstral coefficients [12,13], energy of the segments $[3,14]$, zero-crossing rate $[3,14]$, the autocorrelation function peak $[15,16]$, or harmonic to noise ratio in the sinusoidal model of speech signal [17]. Since each feature has its own disadvantages, new methods tend to use a combination of features for v/uv decision [2,3] and since the value of these features are different for variety of speeches, adaptive thresholding have been used in most of the methods.

Different methods have been used in the field of multifeature voicing decision. Atal and Rabiner [3] clustered the segments into two major groups based on a weighted Euclidian distance in the feature vector space while the weight was estimated according to some statistical properties and the features were considered gaussian distributed in each cluster. Siegel [18] used a non-statistical nonparametric classifier to make v/uv decision. In this method no assumptions are made about the distribution of the features, and training focuses on patterns near the boundry between two regions in the feature space, rather than using statistics to describe each class. Siegel and Bessey [19] tried to develop the mentioned methods by using linear discrimination in the feature vector space.

The use of two or more features in the voicing decision tended to the methods which do not consider the features as a vector and use the best feature for each frame like the work in [20]. The work presented here is in this category. In this work, which is preliminarily presented in [21], we use implicitly two thresholds for each feature and make the decision for each frame based on only one of the features that performs better than other features. This paper is organized as follows. The description of the suggested algorithm is given in Section 2. Some discussions about the new method are presented in Section 3. In Section 4, the results of the algorithm are represented. Section 5 discusses the disadvantages of former methods. Finally, the conclusion is given in Section 6.

\section{The Proposed Algorithm}

In this section we will describe our method for V/UV decision. Here we use three features which are the cepstral peak, autocorrelation function (ACF) peak and zero crossing rate. The speech signal, sampled at $8 \mathrm{kHz}$, is analyzed at $10 \mathrm{~ms}$ intervals using a $40 \mathrm{~ms}$ Hamming window. Then 
the following features are extracted and analyzed.

1) Cepstral peaks: The cepstrum, defined as the real part of the inverse Fourier transform of the log-power spectrum, has a strong peak corresponding to the pitch period of the voiced speech segment being analyzed [22]. Here we use a primary normalization to have a fair decision for all of the frames (including high energy and low energy frames). A 512-point fast Fourier transform (FFT) is used and the peak picking scheme is to determine the cepstral peak in the interval [2.5 - $15 \mathrm{~ms}$ ], corresponding to pitch frequencies between $60-400 \mathrm{~Hz}$. Since the cepstral peaks decrease in amplitude with increasing quefrency, a linear cepstral weight is applied over the 2.5 to $15 \mathrm{~ms}$. The linear cepstral weighting with range of one to five was found empirically by using periodic pulse trains with varying periods as the input to the program.

2) Zero crossing rate: the method in this part is the well known method using the formula (1).

$$
Z_{C} R_{i}=\sum_{n=1}^{N-1}\left|\operatorname{sgn}\left[x_{i}(n)\right]-\operatorname{sgn}\left[x_{i}(n-1)\right]\right|
$$

It is known than that the ZCR of an unvoiced segment is much more than that of a voiced segment.

3) Auto-correlation function peaks: As we know, the speech signal is periodic for voiced segments. So, we make the V/UV decision based on finding a high peak in this function. But since in this algorithm, this function should behave fairly for different segments, it should be normalized, like the cepstral peaks. But, since the speech signal is originally quasi-periodic at voiced segments and the noise of the environment is added, the voiced segments are not precisely periodic. This non-periodicity emerges in the segments with low energy (because SNR falls down) or in high frequencies (since noise is masked in low frequencies but can be destructive in high frequentcies). To eliminate the first effect, we use the method of Center clipping [10], with the clipper's amplitude of $1 / 3$ of the maximum of the absolute of the signal's amplitude. To eliminate the second one, we use a band-pass filter with the cut-off frequencies of 20 and $900 \mathrm{~Hz}$.

After determining how each feature is extracted, we go back to the algorithm. Here the V/UV decision is made in this way: each of the information groups (that obtained from features: Cepstral peaks, ACF peaks and zero crossing rate) is clustered into three clusters by K-Means algorithm [23]. So after clustering, we have three clusters for each feature: the first cluster contains the frames in which the related feature has low values, the second one contains the frames in which the related feature has average values and the third one contains the frames in which the related feature has high values. For example, the frames in the first cluster of zero crossing have low ZCR and so, are very likely to be voiced, despite the third cluster that are very likely to be unvoiced and about the second cluster we cannot conclude yet. But when we consider the three features simultaneously, we can decide about almost all of the frames. It is practically observed that very little frames are found to be in the second cluster for all three features (about $4 \%$ of all the frames). We make the V/UV decision for these frames by clustering them into two clusters, based on autocorrelation function (since it works better than the other features as we will see). Now the only thing remained to do is to decide what to do about the frames that are voiced in a feature and unvoiced in another feature. Here, priority gets important. It means we decide the V/UV of a frame after giving each cluster a priority. The six rules that we choose to determine the priorities are as below:

If a frame belongs to the first cluster of zero-crossing rate, it is voiced.

If a frame belongs to the third cluster of zero-crossing rate, it is unvoiced.

If a frame belongs to the first cluster of cepstral peaks, it is unvoiced.

If a frame belongs to the third cluster of cepstral peaks, it is voiced.

If a frame belongs to the first cluster of ACF peaks, it is voiced.

If a frame belongs to the third cluster of ACF peaks, it is unvoiced.

How these rules are given priorities, is described below:

First, for each rule we calculated the error probability, and the one with the least error probability was chosen as the first priority. Then, to choose the second priority, we calculated the conditional error probability for the rest of the rules, on the condition that the first priority is defined and classifies some frames as voiced or unvoiced (based on which rule is considered as the first priority). For these tests, we used some TIMIT files. We continued in this way until all priorities are defined. The results are as below:

The 1st priority: the third cluster of ACF peaks.

The 2nd priority: the third cluster of cepstral peaks.

The 3rd priority: the third cluster of zero-crossing rate.

The 4th priority: the first cluster of zero-crossing rate.

The 5th priority: the first cluster of ACF peaks.

The 6th priority: the first cluster of cepstral peaks.

Also you will see the complete results with the error probabilities in the simulation and evaluation section.

To improve the performance of the clustering algorithm, we used a limiter for each feature. The reason is that some frames have large values (e.g. ACF peaks) and this causes the clustering algorithm to consider them as a separate cluster in the third cluster. The upper bound for each feature is chosen proportional to the mean of that 
feature in all frames. Also, in order to choose a better upper bound, we eliminated the silence of the start and end of the speech. At last, a median filter of order 5 is found empirically to work well for the resulting V/UV estimates. The block diagram of the proposed algorithm is depicted in Figure 1.

\section{Discussion}

The reason of using three features with three clusters is that each feature will perform well and accurately, just when the value of that feature is very high or very low. So the use of three clusters will help us in this matter. Also to complete the decision, it is necessary to use more than one feature. In this case each feature will correct some of the other features' mistakes, because each feature's base is different from the other one.

Also three clusters can be considered as using two thresholds (similar to double thresholding in the detection topics [24]). So it is obvious that using more than one feature with each having two thresholds will perform better than using some features with one threshold and that will work better than using one feature with one threshold, which is usually used to make a V/UV decision.

The deficiencies of different methods and different features in V/UV decision are discussed below and we will show the deficiencies and faults of each feature with some practical samples.

In the cepstral domain, the considerations and tests' results show that the cepstral peak does not perform well when the signal has limited bandwidth. Inspite of that, when the signal has high frequency coefficients, the cepstral peak is a good indicator of being voiced/ unvoiced. The empirical results that show this can be seen in Figures 2 and 3.

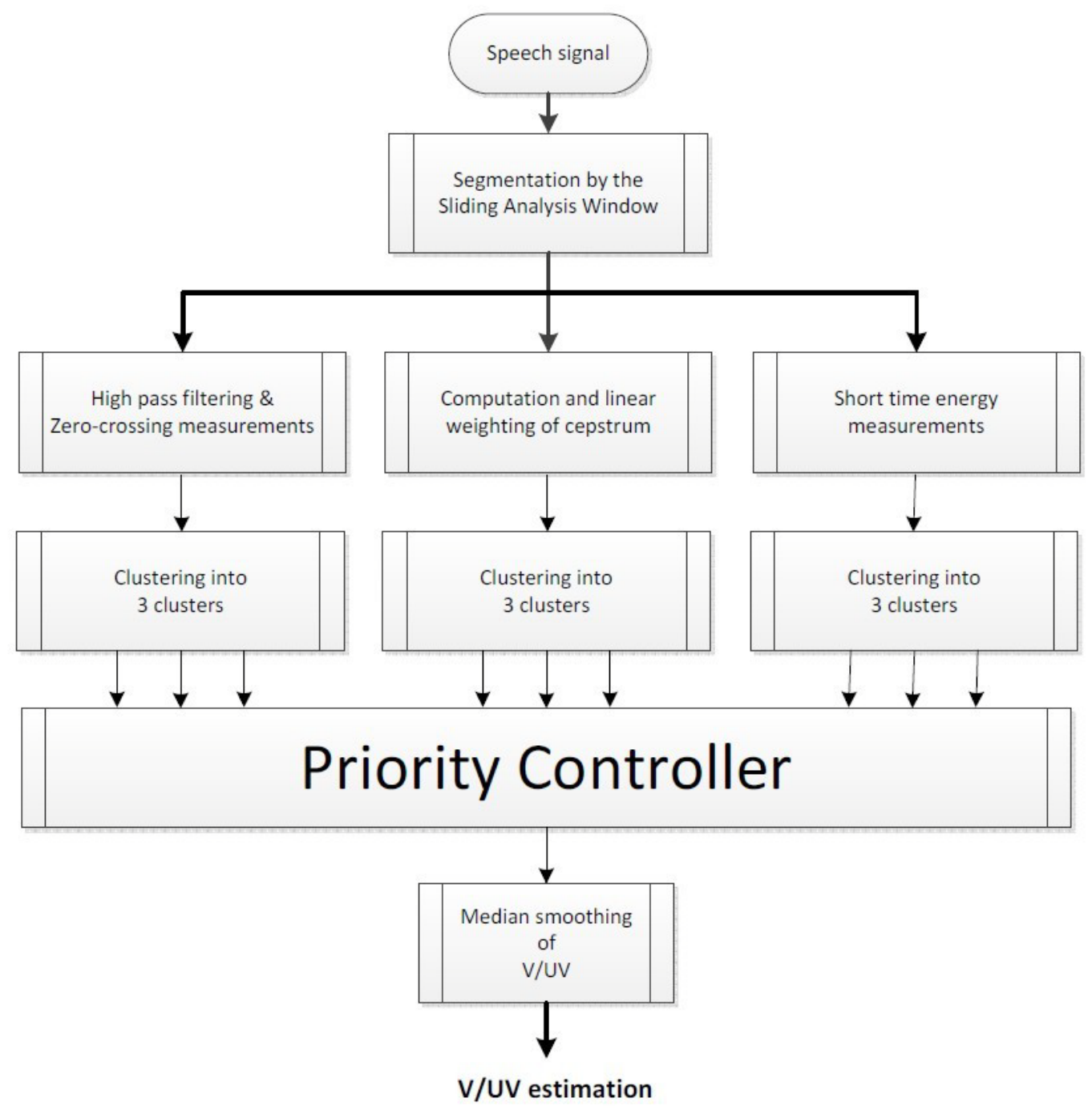

Figure 1. The block diagram of the proposed algorithm. 


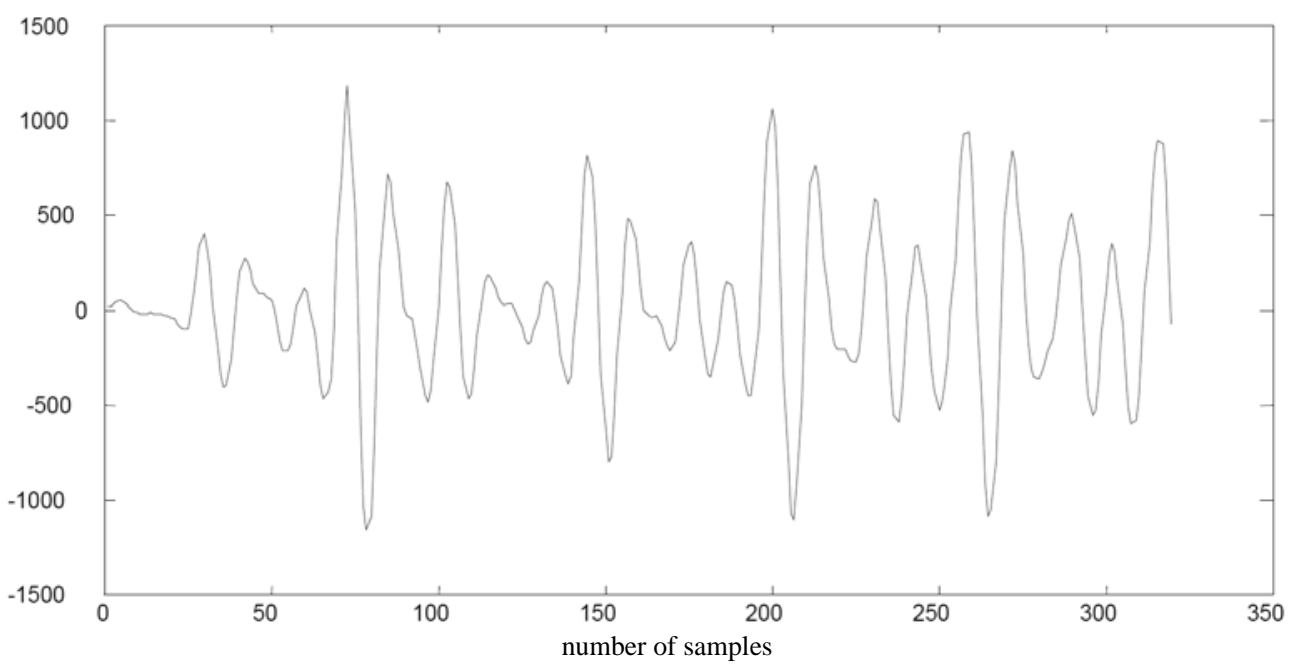

(a)

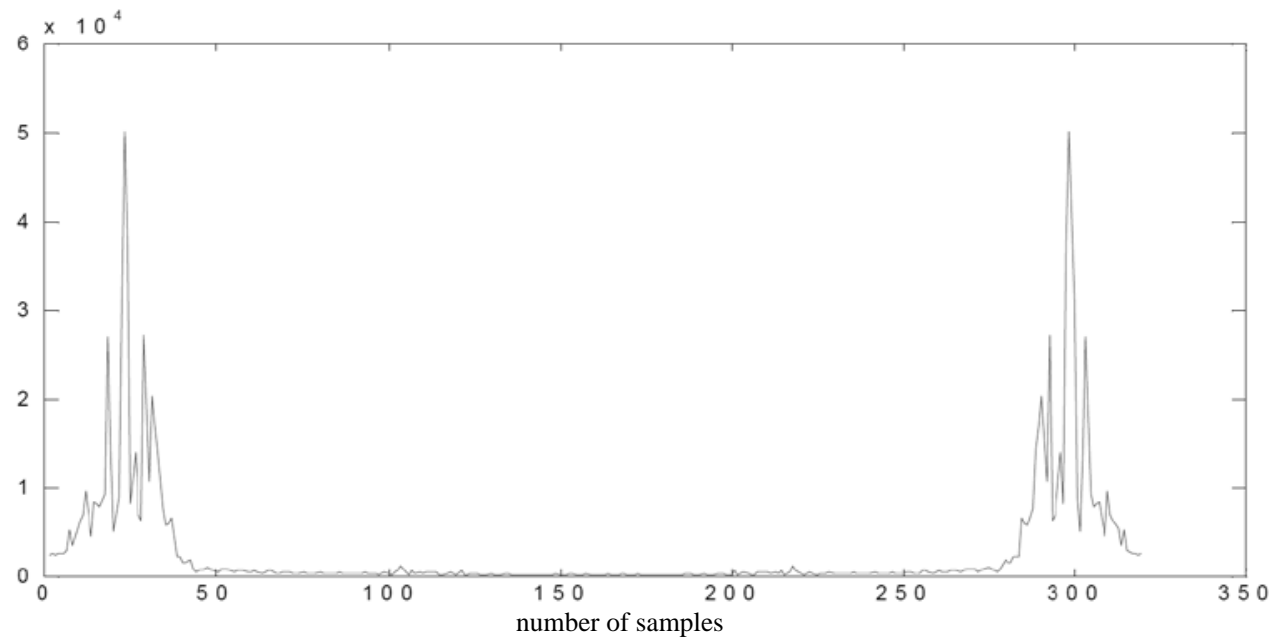

(b)

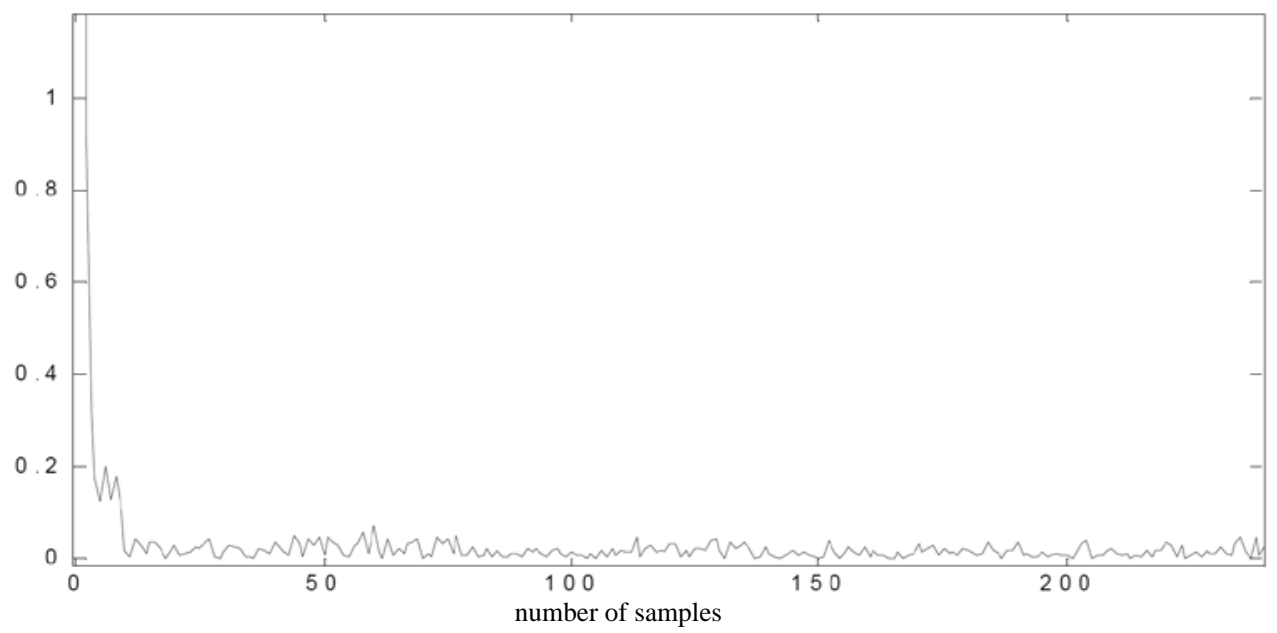

(c)

Figure 2. Testing the cepstral feature. (a) The signal with limited bandwidth; (b) The spectral domain; (c) The cepstral domain. 


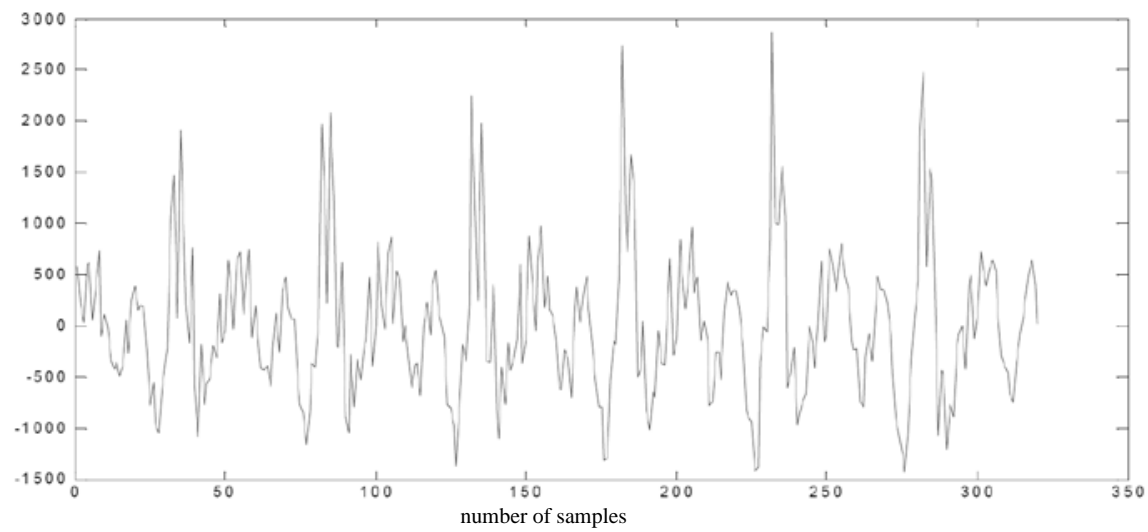

(a)

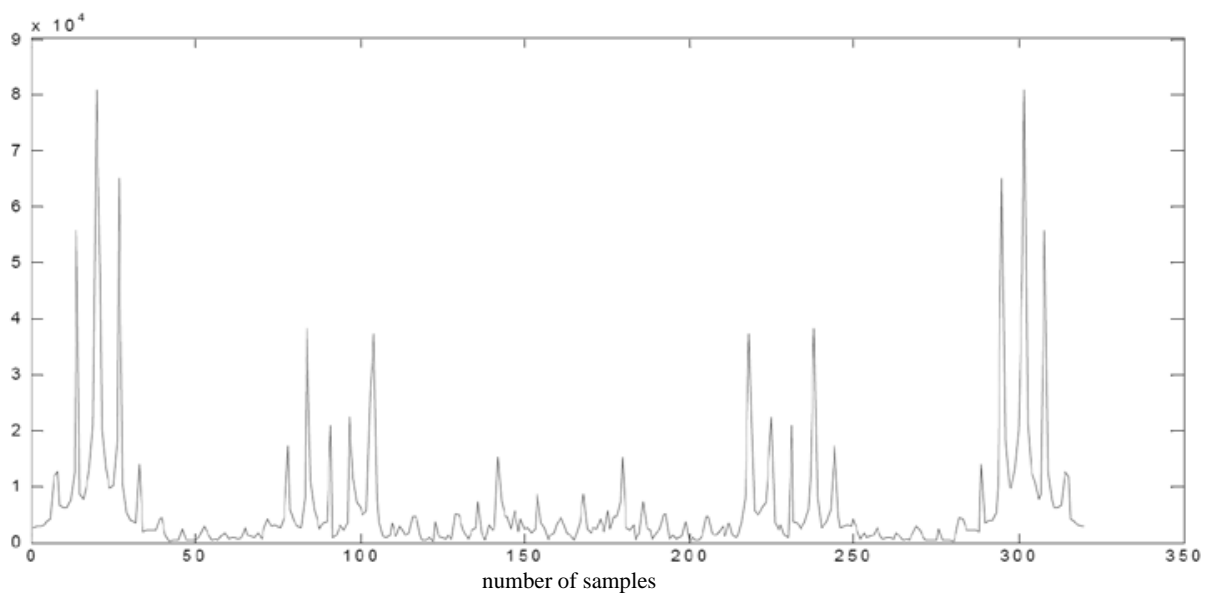

(b)

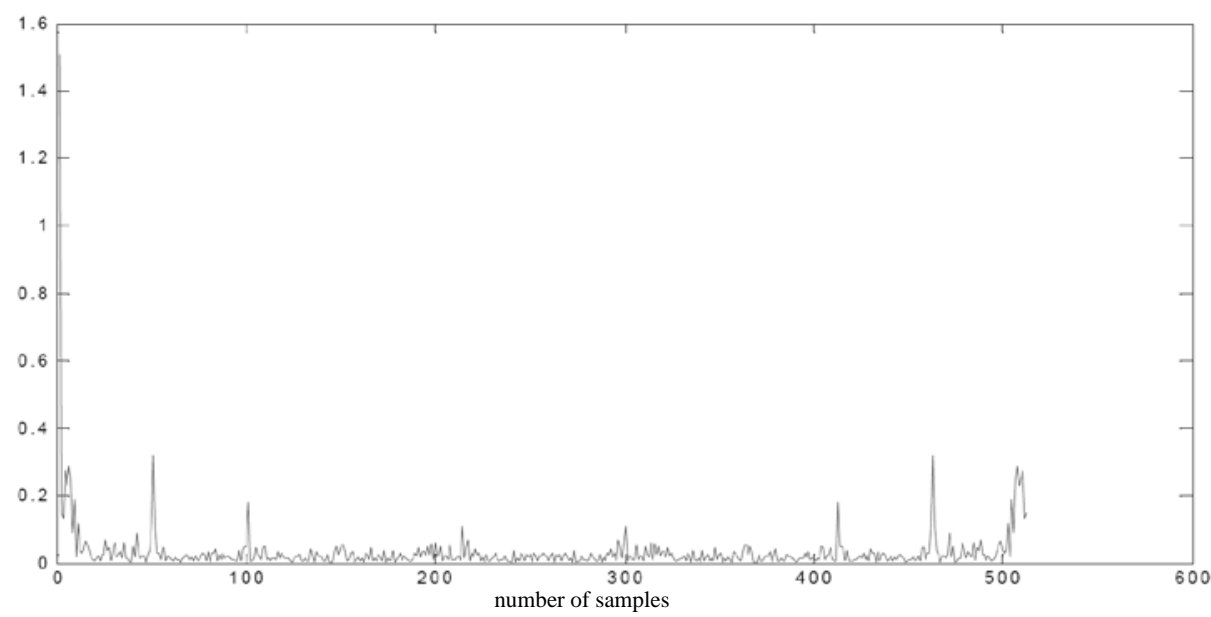

(c)

Figure 3. Testing the cepstral feature. (a) The signal with high frequency coefficients; (b) The spectral domain; (c) The cepstral domain.

Also about the ACF, the effects of the vocal source and vocal tract are convolved with each other in the autocorrelation functions and this results in broad peaks and in some cases multiple peaks in the autocorrelation function [22]. Furthermore, the considerations and tests' results show that the ACF peak does not perform well when the fundamental frequency and the first formant are near each other. This fact can be easily seen in Figure 4. 


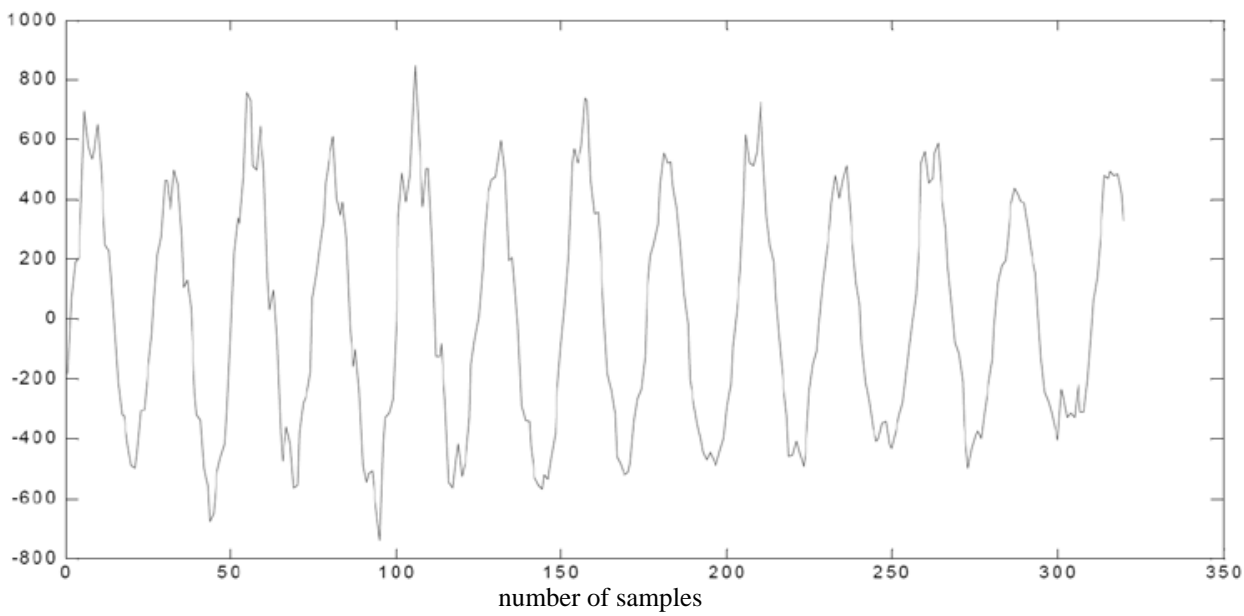

(a)

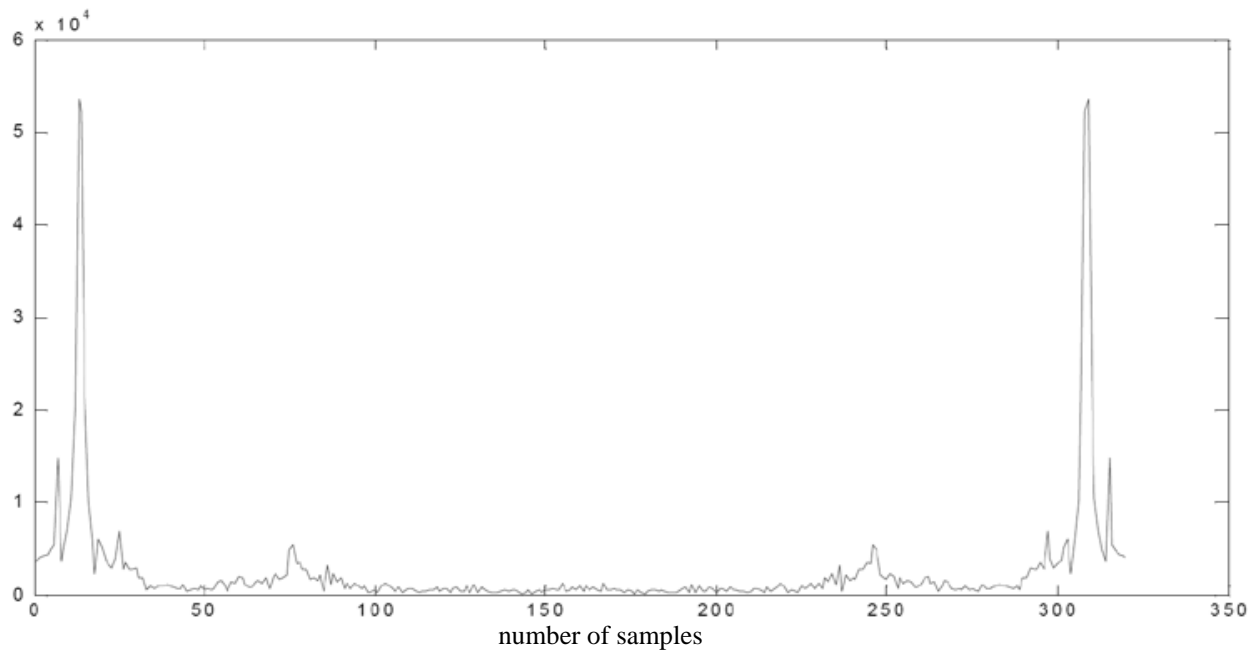

(b)

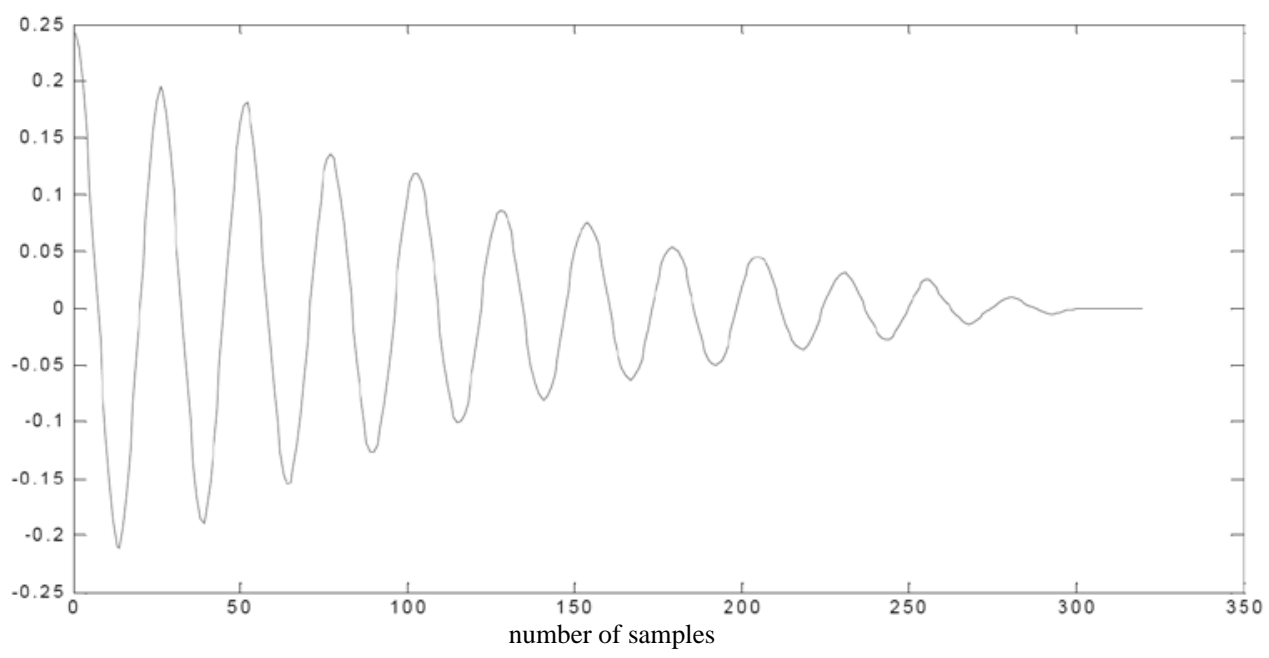

(c)

Figure 4. Testing the ACF feature. (a) A speech signal with the fundamental frequency near the first formant; (b) The spectral domain; (c) The ACF. 
Besides, there are segments in speech that are not periodic but are similar to noise. Inspite of that, their ZCR is low. As an example, the silence segments, between speech, have very low energy but if classified based on their ZCR, they are mistakenly marked voiced. Comparing Figures 5(a) and (b) shows this fact.

In clustering we cluster the values of each feature in all frames into 3 clusters: low, average and high values. In fact, when speech is uttered by a specific person, each feature's values in the voiced segments are very similar to each other. This is true about the unvoiced segments too and this is the base of the clustering method, which performs very well through the tests.

\section{Simulations' Results}

In this section we show the simulations' results and dis- cuss about the quality of the proposed algorithm. 821 frames of speech, that were taken from TIMIT, were tested. To calculate the error probability of each of the rules (the six rules described above with considering the priorities we defined), we counted the number of frames that were classified as voiced or unvoiced in each rule (each priority) based on the priorities we determined. Then we counted the number of frames, which were wrongly classified. The frames were labeled visually by looking at their time domain shape and their frequency domain spectrum. The results are depicted in Table 1. Totally the error for voiced segments was $4.8 \%$ and the error for unvoiced segments was $1.1 \%$.

In the above table etc means the number of frames that are clustered to the second cluster for all three features and are classified as voiced or unvoiced by the clustering

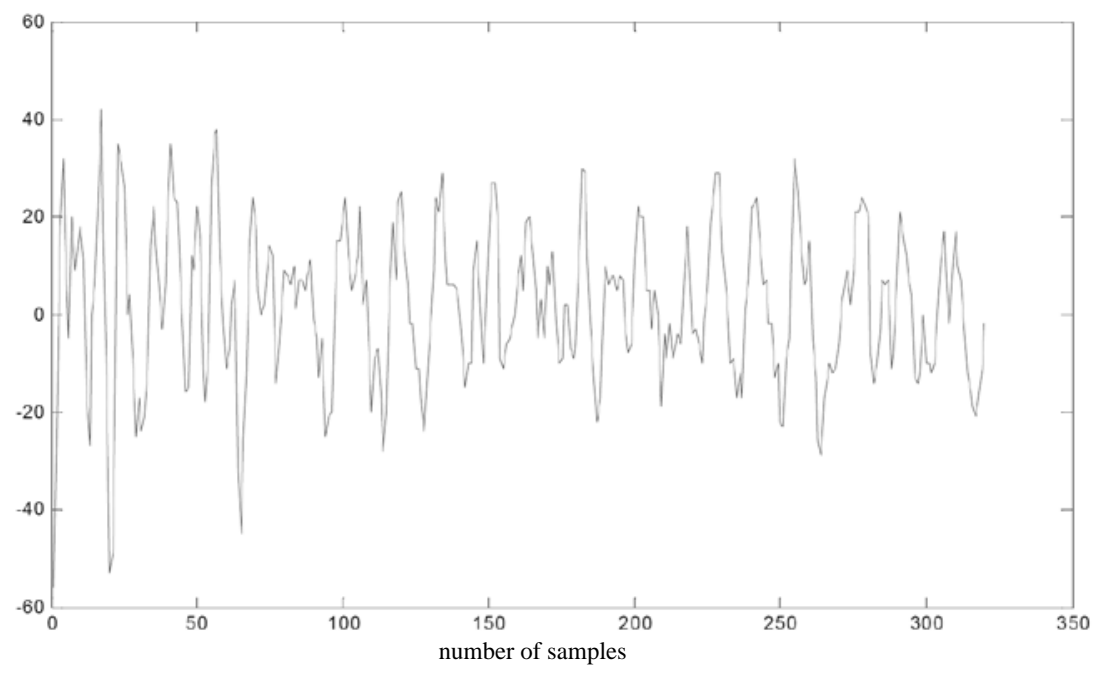

(a)

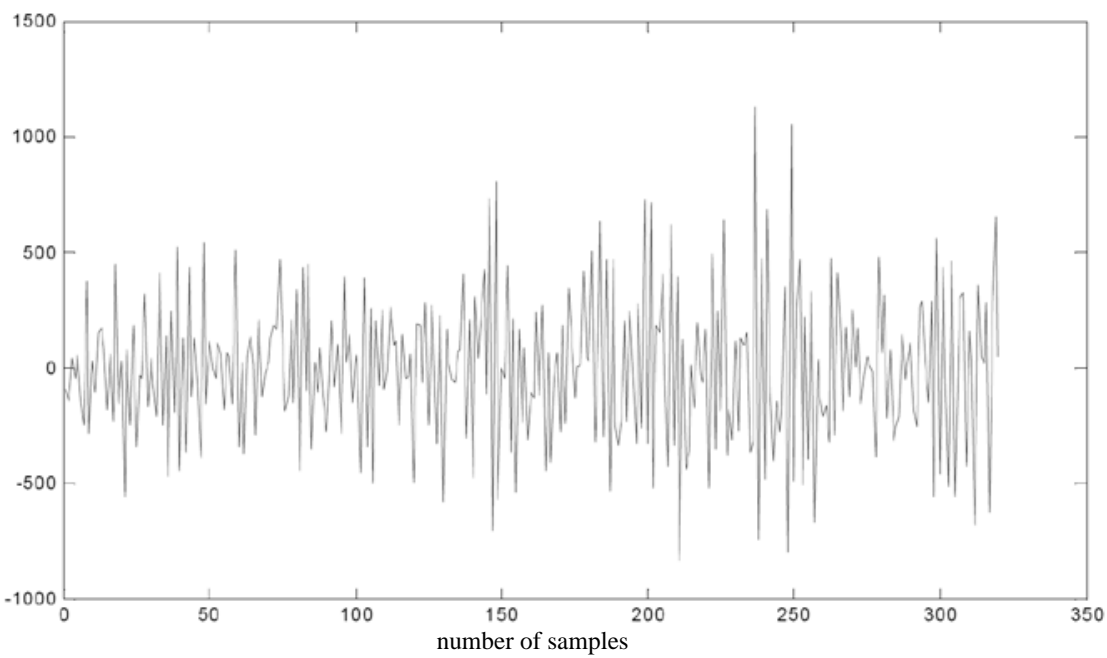

(b)

Figure 5. Testing the ZCR feature. (a) A scilence segment with low energy and low ZCR; (b) An unvoiced segment with high ZCR. 
Table 1. Simulations' results.

\begin{tabular}{|c|c|c|c|c|c|c|c|}
\hline & $\begin{array}{c}\text { auto-3 } \\
\text { (1st priority) }\end{array}$ & $\begin{array}{c}\text { ceps-3 } \\
\text { (2nd priority) }\end{array}$ & $\begin{array}{c}\text { zero-3 } \\
\text { (3rd priority) }\end{array}$ & $\begin{array}{c}\text { zero-1 } \\
\text { (4th priority) }\end{array}$ & $\begin{array}{c}\text { auto-1 } \\
\text { (5th priority) }\end{array}$ & $\begin{array}{c}\text { ceps-1 } \\
\text { (6th priority) }\end{array}$ & etc \\
\hline $\begin{array}{c}\text { The number of } \\
\text { frames identified } \\
\text { V or UV }\end{array}$ & 248 & 46 & 158 & 124 & 142 & 69 & 34 \\
\hline $\begin{array}{l}\text { The number of } \\
\text { frames wrongly } \\
\text { identified }\end{array}$ & 0 & 0 & 1 & 2 & 7 & 10 & 3 \\
\hline
\end{tabular}

method described in section two (clustering to two clusters based on autocorrelation function). To better understand the above table, for example, it shows that $46 \mathrm{fr}-$ ames were clustered in the third cluster of cepstrum (second priority) but not in the third cluster of the autocorrelation function (first priority). So they were classified as voiced and according to the table none of them were misclassified.

\section{Disadvantages of the Cepstrum-Based Voicing Detector}

One of the most common methods for extracting pitch period is to determine the place of the peak in the cepstral domain [10]. Furthermore, the cepstral domain information is used to extract other acoustic parameters. One of these important parameters is "voicing" which is extracted based on the peak value in the cepstral domain by using different methods. But the first problem is that the peak value in the cepstral domain depends on its place on the cepstral axis. So when the pitch period gets larger, the peak value descends with rate $1 / n$. The solution is to multiply a ramp function in the cepstral domain. More details are presented in [20]. More experiments have shown that the cepstral method has other deficiencies. It means that for some voiced frames, although there is a distinguishable peak in the cepstral domain, it does not have sufficient value in comparison with the threshold.

The frames for which cepstrum method cannot perform well can be divided into two categories. For each category a sample frame from TIMIT directory is analyzed which shows the deficiency of the method obviously. Note that both autocorrelation and cepstrum methods are used after the energy-normalization of the frame.

In the categories, in order to prove our claim about all the frames in the group, we model the category with some known mathematical functions such as "sine" and "sinc". The reason that the function can simulate nearly all the frames in the category is discussed in related sections.

The first category contains the vowels which are band-limited in the spectral domain. In this case the cepstral peak value is small and this leads to wrong v/uv classification in the cepstrum-based methods. To prove our claim, we consider a periodic "sinc" function as the input of the cepstrum method. Note that the spectral peak values for this input are the same as each other and if we want to have a spectral shape similar to the frames in this category, we need to multiply this function with some formant-like function in the spectral domain. As it is known, because of the logarithmic property of cepstrum, this multiplication in the spectral domain will result in addition in the cepstral domain and since the periodicity information is in the periodic sinc function, the result of cepstrum method for the periodic sinc function will be similar to the result of applying the method to any frame in this category. Figuers 6 and $\mathbf{7}$ shows the results of this application to two different sincs, one with limited bandwidth and the other with high frequency coefficients.

It can easily be seen that by increasing the bandwidth of the signal the value of the cepstrum feature has increased from 3.69 to 6 .

For more support of our claim we have plotted the value of the cepstrum feature by increasing the bandwidth for a periodic sinc function. The result is depicted in Figure 8. It can be seen that this value increases as the bandwidth increases, meaning that the cepstrum performs better.

The similar results for applying the method to a practical frame of a vowel (/i:/ like in sheet) are shown in Figure 9.

The reason can be explained mathematically for a periodic sinc function (which is an indicator of a band limited signal) as this:

The cepstrum is evaluated from the Equation (2) [10]:

$$
c_{s}(n)=\mathcal{F}^{-1} \log |\mathcal{F}\{s(n)\}|
$$

As we know if $s(n)$ is a periodic sinc, the fft of its absolute value will be the multiplication of a pulse with a delta train. Then, its log will also contain some deltas (the deltas within the pulse width). The larger the sinc's BW is (in other words, the sharper the sinc is) the more deltas will be included in the pulse width (and therefore more deltas we will have at the output of the $\mathrm{ft}$ ). Considering that these deltas show the periodicity of the original waveform (sinc), by increasing the BW, the output of the ifft (in the cepstrum equation) will have larger value at the pitch. 
The second category contains frames related to nasals such as / $\mathrm{n} /$ and / $\mathrm{m} /$ which must be labeled voiced in a correct voicing decision. But the theoretical and practical results show that their cepstral peak values are so small. For modeling nasals to study them, we choose a sine wave, which is a good indicator of nasals.

A theoretical conclusion similar to the one in the first category can be made here. The results of applying this explicit waveform can be seen in Figure 10.

The results of applying the method to a practical frame (/n/ in background) are also shown in Figure 11.

As can be seen for both vowels and nasals, cepstrum based methods do not perform well to extract the parameters of the speech segment, such as voicing and pitch. That's why we do not rely on just one feature. Also we add a third group for each feature (besides the voiced

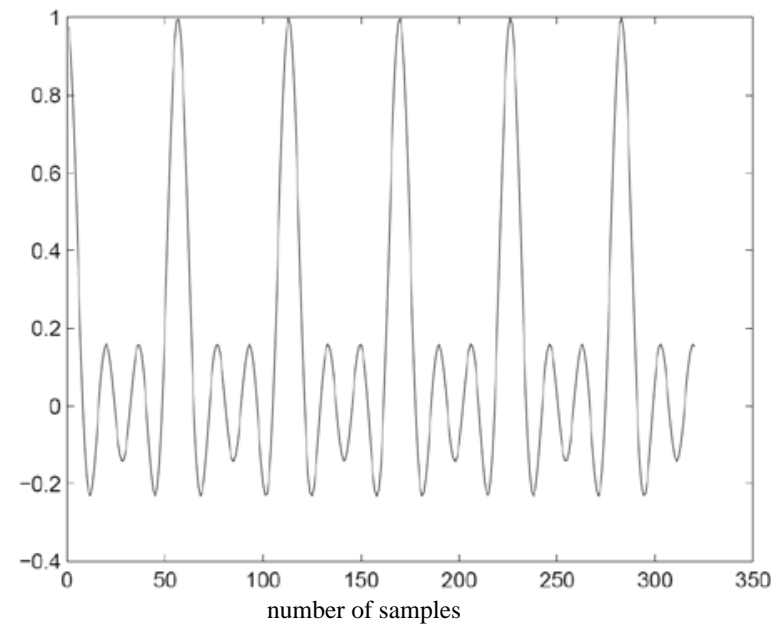

(a) and unvoiced groups), so that if that feature is weak in making the decision, we can go through other features.

\section{Conclusions}

We have presented a new approach of detecting voiced and unvoiced speech. The main advantage of this clustering-based method is getting rid of determining a threshold. So it is highly speaker independent. Also, the use of three features has enabled the method to make a better decision about the segments, in which one feature does not indicate voicing well. Besides, clustering into three clusters, or implicitly, double thresholding, helps us to make the v/uv decision more certainly. Despite the simplicity of the algorithm, the results have shown a satisfactory performance in comparison with more complicated methods.

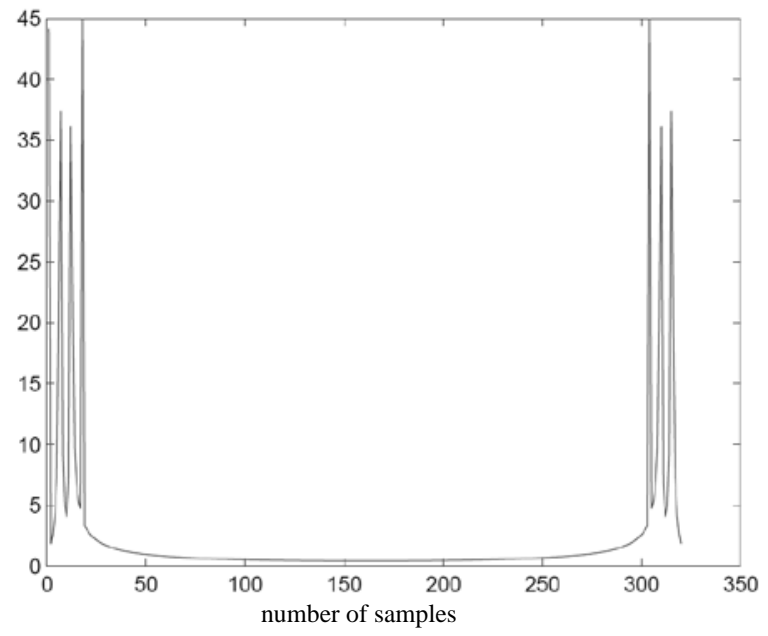

(b)

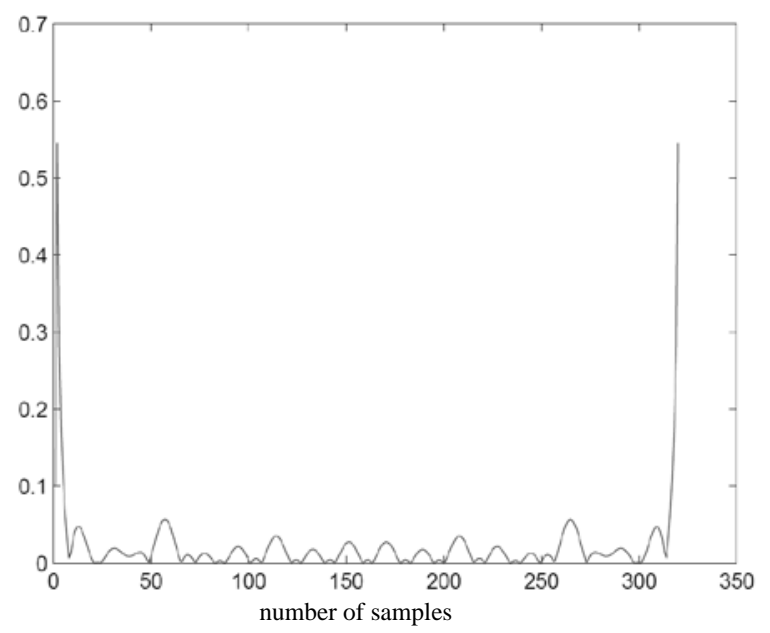

(c)

Figure 6. Testing the sinc function for the first category. (a) A sample sinc function with limited bandwidth; (b) The spectral domain; (c) The cepstral domain, peak to average = 3.69 . 


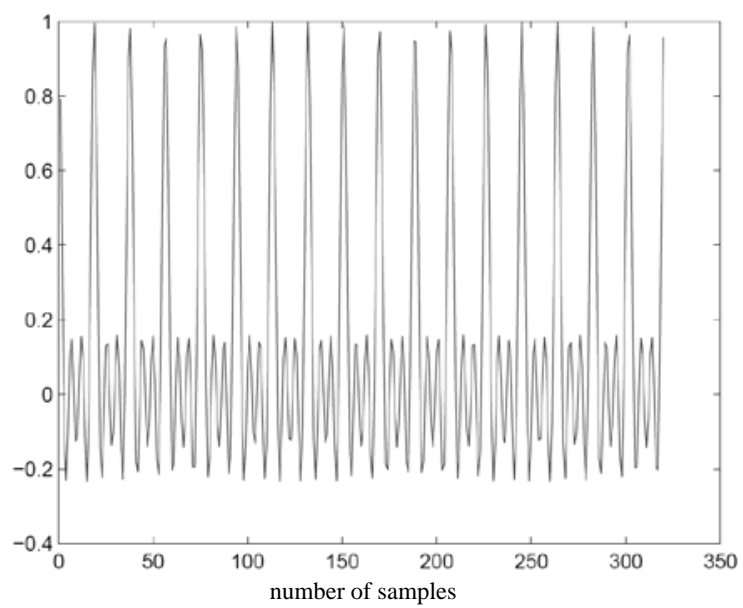

(a)

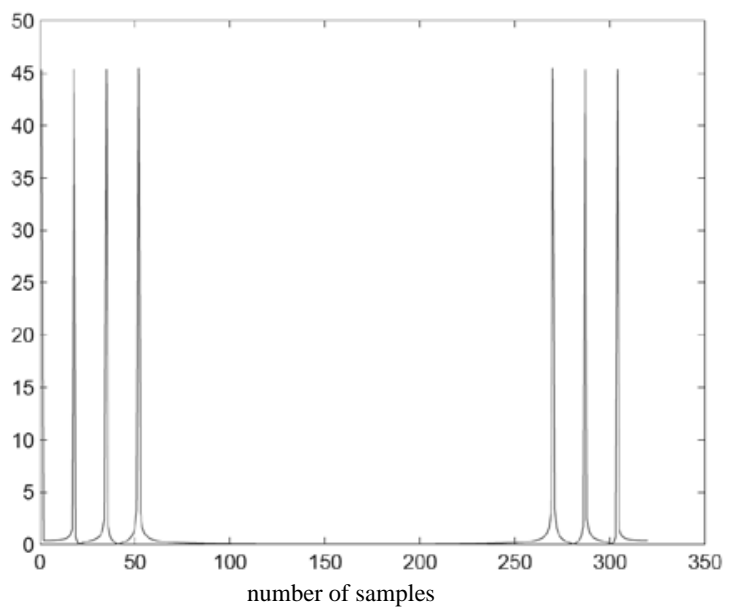

(b)

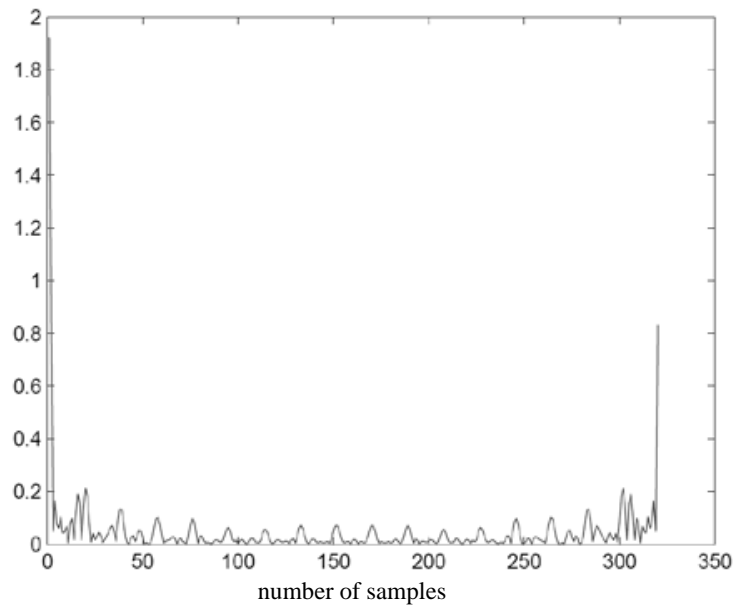

(c)

Figure 7. Testing the sinc function for the first category. (a) A sample sinc function with high frequency coefficients; (b) The spectral domain; (c) The cepstral domain, peak to average $=6$.

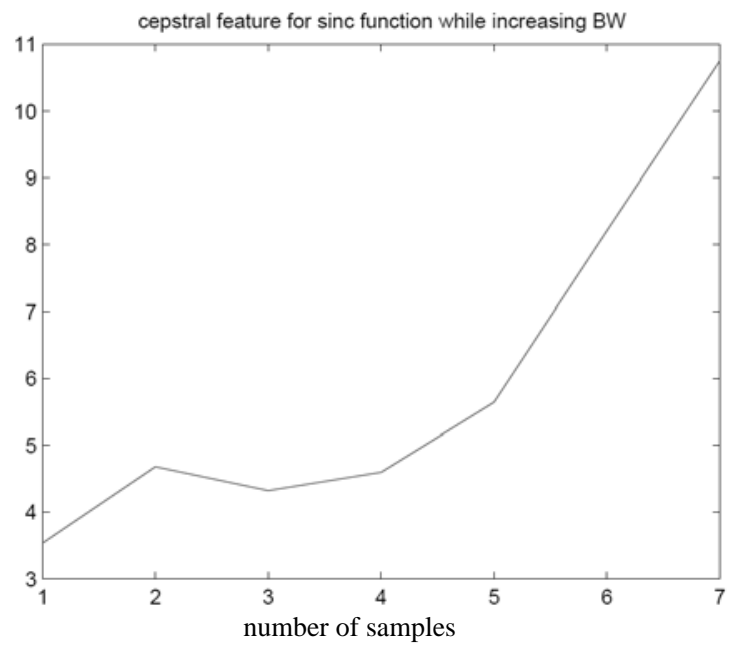

Figure 8. Cepstral feature for the sinc function when increasing BW. 


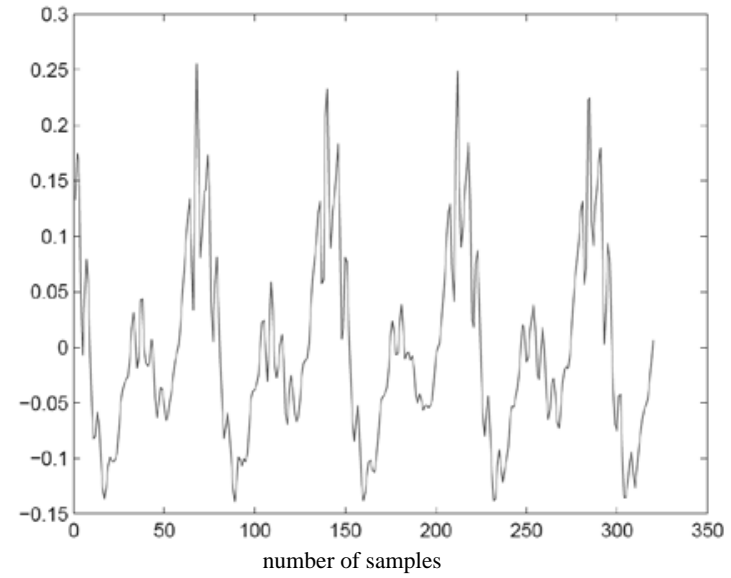

(a)

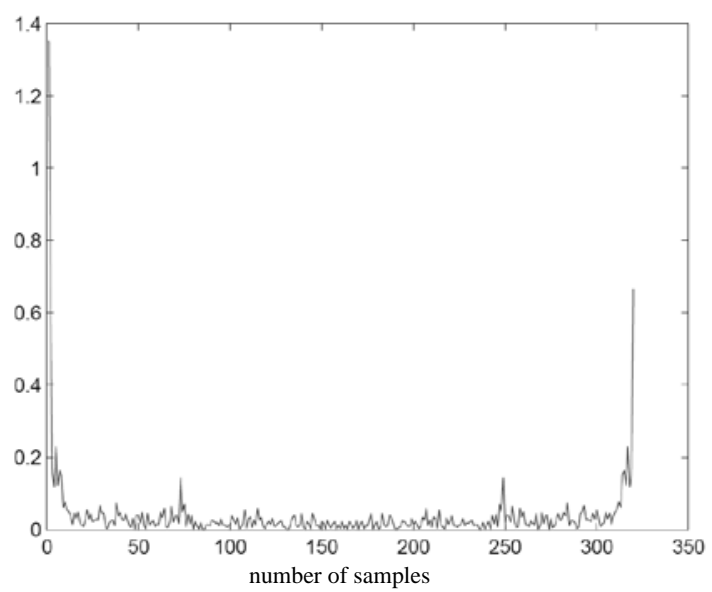

(b)

Figure 9. Testing a real speech frame for the first category. (a) The vowel /i:/ as in sheet; (b) The cepstral domain.

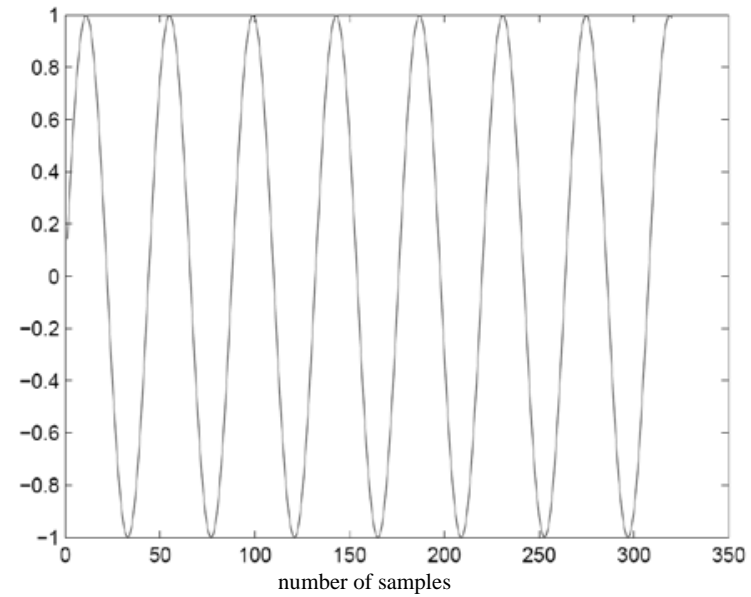

(a)

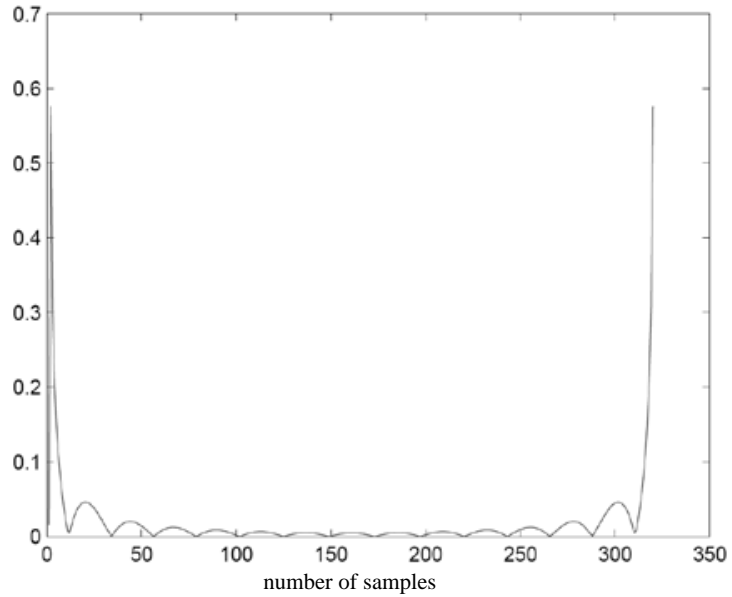

(b)

Figure 10. Testing a sample sine function. (a) The sample sine function; (b) The cepstral domain.

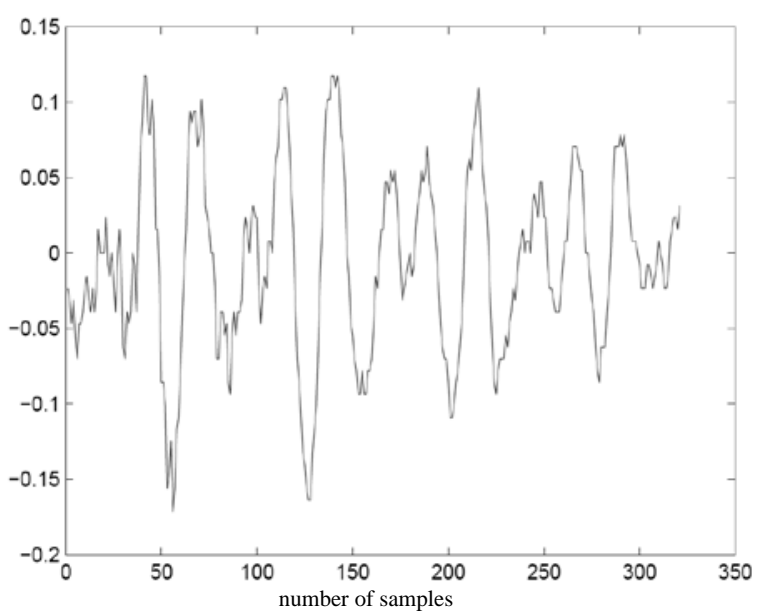

(a)

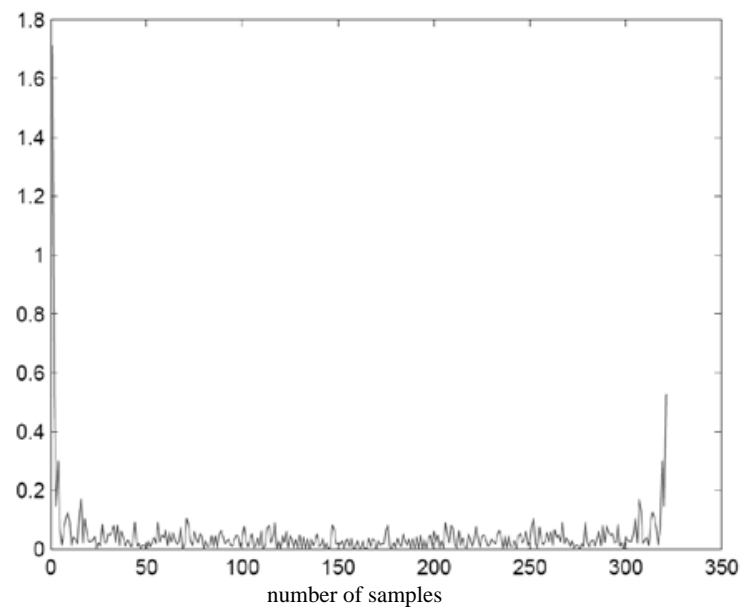

(b)

Figure 11. Testing a real speech frame for the second category. (a) The nasal /n/; (b) The cepstral domain. 


\section{REFERENCES}

[1] E. Fisher, J. Tabrikian and S. Dubnov, "Generalized Likelihood Ratio Test for Voiced-Unvoiced Decision in Noisy Speech Using the Harmonic Model," IEEE Transactions on Audio, Speech, and Language Processing, Vol. 14, No. 2, 2006, pp. 502-510. doi:10.1109/TSA.2005.857806

[2] Y. Qi and B. R. Hunt, "Voiced-Unvoiced-Silence Classifications of Speech Using Hybrid Features and a Network Classifier," IEEE Transactions on Speech and Audio Processing, Vol. 1, No. 2, 2002, pp. 250-255. doi:10.1109/89.222883

[3] B. Atal and L. Rabiner, "A Pattern Recognition Approach to Voicedunvoiced-Silence Classification with Applications to Speech Recognition,” IEEE Transactions on Acoustics, Speech and Signal Processing, Vol. 24, No. 3, 2003, pp. 201-212. doi:10.1109/TASSP.1976.1162800

[4] F. Y. Qi and C. C. Bao, "A Method for Voiced/Unvoiced/Silence Classification of Speech with Noise Using SVM," Acta Electronica Sinica, Vol. 34, No. 4, 2006, pp. 605-611.

[5] P. Jancovic and M. Kokuer, "Estimation of VoicingCharacter of Speech Spectra Based on Spectral Shape," IEEE Signal Processing Letters, Vol. 14, No. 1, 2006, pp. 66-69. doi:10.1109/LSP.2006.881517

[6] B. Atal and M. Schroeder, "Predictive Coding of Speech Signals and Subjective Error Criteria," IEEE Transactions on Acoustics, Speech and Signal Processing, Vol. 27, No. 3, 2003, pp. 247-254. doi:10.1109/TASSP.1979.1163237

[7] L. Hui, B. Dai and L. Wei, “A Pitch Detection Algorithm Based on AMDF and ACF," 2006 IEEE International Conference on Acoustics, Speech and Signal Processing, Toulouse, 14-19 May 2006.

[8] P. A. Naylor, A. Kounoudes, J. Gudnason and M. Brookes, "Estimation of Glottal Closure Instants in Voiced Speech Using the DYPSA Algorithm," IEEE Transactions on Audio, Speech and Language Processing, Vol. 15, No. 1, 2007, pp. 34-43. doi:10.1109/TASL.2006.876878

[9] A. V. Oppenheim, "Speech Spectrograms Using the Fast Fourier Transform,” IEEE Spectrum, Vol. 7, No. 8, 2009, pp. 57-62. doi:10.1109/MSPEC.1970.5213512

[10] J. R. Deller, J. G. Proakis and J. H. L. Hansen, "DiscreteTime Processing of Speech Signals,” 2nd Edition, IEEE Press, New York, 2000.

[11] Z. D. Zhao, X. M. Hu and J. F. Tian, “An Effective Pitch Detection Method for Speech Signals with Low Signal-to-Noise Ratio," International Conference on Machine Learning and Cybernetics, Vol. 5, 2008, pp. 27752778,.

[12] S. Imai, "Cepstral Analysis Synthesis on the Mel Frequency Scale," IEEE International Conference on Acoustics, Speech, and Signal Processing, Vol. 8, 2003, pp.
93-96.

[13] J. K. Shah, A. N. Iyer, B. Y. Smolenski and R. E. Yantorno, "Robust Voiced/Unvoiced Classification Using Novel Features and Gaussian Mixture Model," IEEE International Conference on Acoustics, Speech, and Signal Processing, Philadelphia, 2004, pp. 17-21.

[14] R. G. Bachu, S. Kopparthi, B. Adapa and B. D. Barkana, "Separation of Voiced and Unvoiced Using Zero Crossing Rate and Energy of the Speech Signal," American Society for Engineering Education (ASEE) Zone Conference Proceedings, 2008, pp. 1-7.

[15] L. Rabiner, "On the Use of Autocorrelation Analysis for Pitch Detection,” IEEE Transactions on Acoustics, Speech and Signal Processing, Vol. 25, No. 1, 2003. pp. 24-33. doi:10.1109/TASSP.1977.1162905

[16] M. S. Rahman and T. Shimamura, "Pitch Determination Using Autocorrelation Function in Spectral Domain," Eleventh Annual Conference of the International Speech Communication Association, Makuhari, 2010, pp. 653656.

[17] R. J. McAulay and T. F. Quatieri, "Pitch Estimation and Voicing Detection Based on a Sinusoidal Speech Model," International Conference on Acoustics, Speech, and Signal Processing, Vol. 1, 1990, pp. 249-252. doi:10.1109/ICASSP.1990.115585

[18] L. Siegel, "A Procedure for Using Pattern Classification Techniques to Obtain a Voiced/Unvoiced Classifier,” IEEE Transactions on Acoustics, Speech and Signal Processing, Vol. 27, No. 1, 2003, pp. 83-89. doi:10.1109/TASSP.1979.1163186

[19] L. Siegel and A. Bessey, "Voiced/Unvoiced/Mixed Excitation Classification of Speech," IEEE Transactions on Acoustics, Speech and Signal Processing, Vol. 30, No. 3, 2003, pp. 451-460. doi:10.1109/TASSP.1982.1163910

[20] S. Ahmadi and A. S. Spanias, "Cepstrum-Based Pitch Detection Using a New Statistical V/UV Classification Algorithm," IEEE Transactions on Speech and Audio Processing, Vol. 7, No. 3, 2002, pp. 333-338. doi:10.1109/89.759042

[21] M. Radmard, M. Hadavi, S. Ghaemmaghami and M. M. Nayebi, "Clustering Based Voiced/Unvoiced Decision for Speech Signals,” Signal Processing Symposium (SPS), Poland, 2011.

[22] A. M. Noll, "Clipstrum Pitch Determination,” The Journal of the Acoustical Society of America, Vol. 44, No. 6, 1968, pp. 1585-1591. doi:10.1121/1.1911300

[23] J. A. Hartigan and M. A. Wong, “A K-Means Clustering Algorithm,” Journal of the Royal Statistical Society. Series C, Vol. 28, No. 1, 1979, pp. 100-108.

[24] H. V. Poor, "An Introduction to Signal Detection and Estimation,” Springer, Berlin, 1994. 